\title{
Special lattice computation for the CERN Compact Linear Collider
}

\author{
E. T. d'Amico and G. Guignard \\ CERN, Geneva, Switzerland
}

(Received 12 October 2000; revised manuscript received 7 December 2000; published 19 February 2001)

\begin{abstract}
In the study of a high-energy linear collider such as the CERN Compact Linear Collider (CLIC), there are domains of activities where analytical developments are useful guides before doing fully numerical calculations or using standard accelerator design programs. The purpose of this report is to illustrate the interest of such an approach in designing rarely used magnetic insertions, such as isochronous modules or tunable achromats which are required in several locations of the CLIC complex. Algorithmic codes have been written in this context. They give global information or guidance among a variety of approximated solutions and help the user in selecting the most promising one before fully numerical calculations and, eventually, optimizations are carried out to obtain the solution consistent with the physical model.
\end{abstract}

DOI: 10.1103/PhysRevSTAB.4.021002

\section{INTRODUCTION}

The main injector of the CERN Compact Linear Collider (CLIC) has to be connected to the linear collider through transfer lines which have to be designed carefully to retain the quality of the beam. In particular, the bunch length growth due to the bending loops must be kept to a very low value which means that these lines should be as isochronous as possible, while not causing too large an emission of synchrotron radiation. Moreover, the driver complex which generates the rf power contains a few combiner rings and several transfer lines where the isochronicity or its tuning are of paramount importance. The standard lattice and insertion design programs, such as MAD [1], have difficulty finding stable solutions and need to be started from good approximations. These are usually obtained analytically by considering some or all the magnetic elements as thin magnetic lenses. However, the analytical development generates a large variety of solutions, the choice among which is subject to many parameters. Thus, a program based on this development should be able to help the designer in making the best choice by providing him with the needed clues, and by narrowing interactively the selection in this multiparameter space.

The codes which had to be developed are written mainly in FORTRAN 77 for historical reasons, but they are built in a modular fashion and run in a UNIX environment. Because they call upon large general purpose packages such as MAD, they also involve parts written at the script level.

\section{DESIGN OF SPECIAL LATTICES}

The CLIC study [2] and the new CLIC test facility [3] involve magnetic lattices that are able to modify the length of the beam bunch (isochronicity tuning) or not (isochronicity) and to match them to each other or to a standard FODO line. The complexity of such lattices (rings or transfer lines) makes it very difficult to design them globally. Thus it has been proposed to build them from modules, each matched to the next one. This strategy
PACS numbers: 41.85.-p, 41.75.-i, 29.27. $-\mathrm{a}, 29.17 .+\mathrm{w}$

(analogous to the standard breaking up of a complex computer program into many much simpler routines) proved to be very efficient and fast in treating special lattices. Three of these modules are described hereafter.

\section{A. Isochronous achromat}

It has been shown that the minimum number of deflecting magnets in an isochronous insertion is 3. For reasons of simplicity, the chosen insertion shown in Fig. 1 consists of three bending magnets geometrically and magnetically symmetric around the middle plane of the second magnet [4]. To simplify the algebra, these magnets are treated as sector magnets of equal length $l_{m}$ but different deflection angles $\phi_{1}$ and $\phi_{2}$ for the first and second dipole, respectively. The space between the first two magnets is filled by a drift space of length $L_{1}$, a focusing quadrupole of length $l_{q}$ and normalized gradient $k_{1}$, a second drift space of length $L_{2}$, a defocusing quadrupole of length $l_{q}$ and normalized gradient $k_{2}$, and, finally, a third drift space of length $L_{3}$. On the assumption that the quadrupoles are perfectly centered, the parameter $R_{56}$ is given by

$$
R_{56}=\int_{s_{1}}^{s_{2}} \frac{D_{x}}{\rho(s)} d s
$$

where $D_{x}$ is the horizontal dispersion, $\rho(s)$ is the radius of curvature, and $s_{1}, s_{2}$ are the longitudinal coordinates of the beginning and end of the beam line. The $R_{56}$ parameter is positive if high-momentum particles of the bunch travel longer paths. Assuming also that the dispersion and its

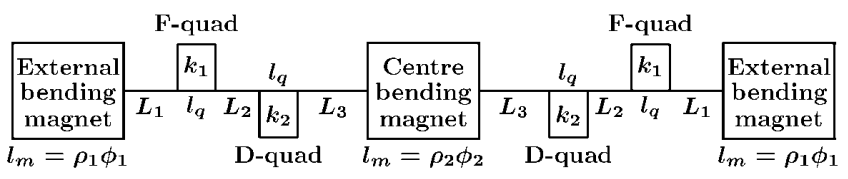

FIG. 1. An isochronous insertion consisting of three bending magnets. 
derivative are zero at the entrance of the first dipole, the contributions of the first dipole and half of the second dipole to this integral are, respectively [4],

$$
\begin{gathered}
\rho_{1}\left(\phi_{1}-\sin \phi_{1}\right), \\
D_{j} \sin \left(\phi_{2} / 2\right)-\rho_{2} D_{j}^{\prime}\left[\cos \left(\phi_{2} / 2\right)-1\right] \\
+\rho_{2}\left[\phi_{2} / 2-\sin \left(\phi_{2} / 2\right)\right],
\end{gathered}
$$

where $\rho_{1}$ and $\rho_{2}$ are the trajectory radii in the first and second dipoles, respectively, and $D_{j}$ and $D_{j}^{\prime}$ are the dispersion and its derivative at the entrance of the second dipole. Thus the following equation is obtained:

$$
\begin{aligned}
\frac{R_{56}}{2}= & \rho_{1}\left(\phi_{1}-\sin \phi_{1}\right)+D_{j} \sin \left(\phi_{2} / 2\right) \\
& -\rho_{2} D_{j}^{\prime}\left[\cos \left(\phi_{2} / 2\right)-1\right] \\
& +\rho_{2}\left[\phi_{2} / 2-\sin \left(\phi_{2} / 2\right)\right] .
\end{aligned}
$$

In order to obtain a nondispersive module, the derivative of the dispersion at the point of symmetry must be zero, providing a second equation,

$$
-\frac{\sin \left(\phi_{2} / 2\right)}{\rho_{2}} D_{j}+D_{j}^{\prime} \cos \left(\phi_{2} / 2\right)+\sin \left(\phi_{2} / 2\right)=0 .
$$

From these two equations it is easy to obtain

$$
\begin{aligned}
D_{j}^{\prime} & =\frac{1}{\rho_{2}}\left[\frac{R_{56}}{2}-l_{m}\left(\frac{3}{2}-\frac{\sin \phi_{1}}{\phi_{1}}\right)\right], \\
D_{j} & =\rho_{2}\left[1+D_{j}^{\prime} \cot \left(\phi_{2} / 2\right)\right] .
\end{aligned}
$$

It is possible to obtain the expressions of the lengths of the first two drift spaces as functions of $k_{1}, k_{2}$ and of $L_{3}$,

$$
\begin{aligned}
& L_{1}=a \frac{C_{2} q_{1}}{C_{1} q_{2}}\left(L_{3}-\frac{D_{j}}{D_{j}^{\prime}}+q_{2}\right)-l+q_{1}, \\
& L_{2}=q_{1}-q_{2}+\frac{b}{L_{3}-\frac{D_{j}}{D_{j}^{\prime}}+q_{2}},
\end{aligned}
$$

where

$$
\begin{gathered}
l=\rho_{1} \tan \left(\phi_{1} / 2\right), \quad a=-\frac{D_{j}^{\prime}}{\sin \phi_{1}}=-\frac{x}{\rho_{2} \sin \phi_{1}} \\
b=\frac{q_{2}}{C_{2}}\left(\frac{q_{2}}{C_{2}}+\frac{q_{1}}{a C_{1}}\right), \quad q_{i}=\frac{C_{i}}{S_{i} \sqrt{k_{i}}}, \\
C_{1}=\cos \left(l_{q} \sqrt{k_{1}}\right), \quad S_{1}=\sin \left(l_{q} \sqrt{k_{1}}\right), \\
C_{2}=\cosh \left(l_{q} \sqrt{k_{2}}\right), \quad S_{2}=\sinh \left(l_{q} \sqrt{k_{2}}\right) .
\end{gathered}
$$

The lengths $L_{1}$ and $L_{2}$ depend on the parameter $R_{56}$ through the quantities $D_{j}$ and $D_{j}^{\prime}$. The insertion is isochronous if $R_{56}=0$. Equations (4) generate a family of insertions according to the actual values of $k_{1}, k_{2}$ and $L_{3}$. Of course, $L_{1}$ and $L_{2}$ must be positive and larger than a specified physical length $\delta$. The algebra to find the valid ranges of $k_{1}, k_{2}$ and $L_{3}$ is tedious and can be found in [4]. The results are given in Table I with the following definitions:

$$
\begin{gathered}
\left.C^{*}=\frac{a q_{1}}{C_{1}\left(l-q_{1}+\delta\right)}, \quad k^{*}=\frac{1}{\left[\delta-q_{1}-\frac{q_{1}^{2}}{C_{1}^{2}\left(l-q_{1}+\delta\right.}\right)}\right]^{2} \\
\frac{\tanh x_{m}}{x_{m}}=\frac{1}{l_{q} \sqrt{k^{*}}-1}, \\
u\left(x_{m}\right)=x_{m} \sinh \left(x_{m}\right)-l_{q} \sqrt{k^{*}}\left[\cosh \left(x_{m}\right)-C^{*}\right], \\
(l+\delta) \sqrt{k_{1}^{(1)}} \tan \left(l_{q} \sqrt{k_{1}^{(1)}}\right)-1=0, \\
{\left[k_{1}^{(2)} \delta(l+\delta)-1\right] \sin \left(l_{q} \sqrt{k_{1}^{(2)}}\right)-\sqrt{k_{1}^{(2)}}(l+2 \delta) \cos \left(l_{q} \sqrt{k_{1}^{(2)}}\right)=0,} \\
(l+\delta) \sqrt{k_{1}^{(3)}} \sin \left(l_{q} \sqrt{k_{1}^{(3)}}\right)-\cos \left(l_{q} \sqrt{k_{1}^{(3)}}\right)-a=0, \\
d \sqrt{k_{1}^{(4)}} \tan \left(l_{q} \sqrt{k_{1}^{(4)}}\right)-1=0, \\
\sqrt{k_{2}^{(1)}} \sinh \left(l_{q} \sqrt{k_{2}^{(1)}}\right)-\sqrt{k^{*}}\left[\cosh \left(l_{q} \sqrt{k_{2}^{(1)}}\right)-C^{*}\right]=0, \\
\sqrt{k_{2}^{(2)}}, \sqrt{k_{2}^{(3)}} \operatorname{are~the~} \operatorname{rots} \text { of the equations: } \\
\sqrt{k_{2}} \sinh \left(l_{q} \sqrt{k_{2}}\right)-\sqrt{k^{*}}\left[\cosh \left(l_{q} \sqrt{k_{2}}\right)-C^{*}\right]=0 \quad \text { with } \sqrt{k^{*}}>2 / l_{q} \quad \text { and } \quad C^{*}>1, \\
\sqrt{k_{2}^{(4)}} \sinh \left(l_{q} \sqrt{k_{2}^{(4)}}\right)+\sqrt{k^{*}}\left[\cosh \left(l_{q} \sqrt{k_{2}^{(4)}}\right)-C^{*}\right]=0, \\
L_{3}^{(1)}=L_{3}^{(0)}+\frac{b}{\delta+q_{2}-q_{1}}, \quad L_{3}^{(2)}=L_{3}^{(0)}+\frac{q_{2} C_{1}}{a q_{1} C_{2}}\left(l-q_{1}+\delta\right) .
\end{gathered}
$$

An example of an isochronous arc is shown in Fig. 2, which displays the optics functions of the quarter part of a combiner ring in the preliminary design of the CLIC rf power source. 
TABLE I. Valid ranges of $k_{1}, k_{2}$, and $L_{3}$.

\begin{tabular}{ccc}
\hline \hline$k_{1}$ & $k_{2}$ & $L_{3}$ \\
\hline$k_{1} \leq k_{1}^{(1)}$ & $q_{2}<q_{1}-\delta$ & $q_{2}>q_{1}-\delta$ \\
$k_{1}^{(3)} \leq k_{1}<k_{1}^{(2)}$ & $k_{2}>k_{2}^{(1)}$ & $L_{3}^{(0)}<L_{3}^{(0)}<L_{3}^{(1)}$ \\
$k_{1}^{(1)}<k_{1}<\min \left\{k_{1}^{(2)}, k_{1}^{(3)}\right\}$ & & $L_{3}^{(2)}<L_{3}<L_{3}^{(1)}$ \\
$l_{q} \sqrt{k^{*}} \leq 2$ or & $k_{2}>0$ & \\
$l_{q} \sqrt{k^{*}}>2$ and $u\left(x_{m}\right) \geq 0$ & & $L_{3}^{(2)}<L_{3}<L_{3}^{(1)}$ \\
$k_{1}^{(1)}<k_{1}<\min \left\{k_{1}^{(2)}, k_{1}^{(3)}\right\}$ & $0<k_{2}<k_{2}^{(2)}$ or & \\
$l_{q} \sqrt{k^{*}}>2$ and $u\left(x_{m}\right)<0$ & $k_{2}>k_{2}^{(3)}$ & $L_{3}^{(2)}<L_{3}<L_{3}^{(1)}$ \\
$k_{1}^{(2)}<k_{1}<k_{1}^{(3)}$ & $k_{2}<k_{2}^{(4)}$ & $L_{3}^{(2)}<L_{3}<L_{3}^{(1)}$ \\
$k_{1}^{(1)}<k_{1}<k_{1}^{(4)}$ & $q_{2}<q_{1}-\delta$ & $L_{3}>L_{3}^{(2)}$ \\
\hline \hline
\end{tabular}
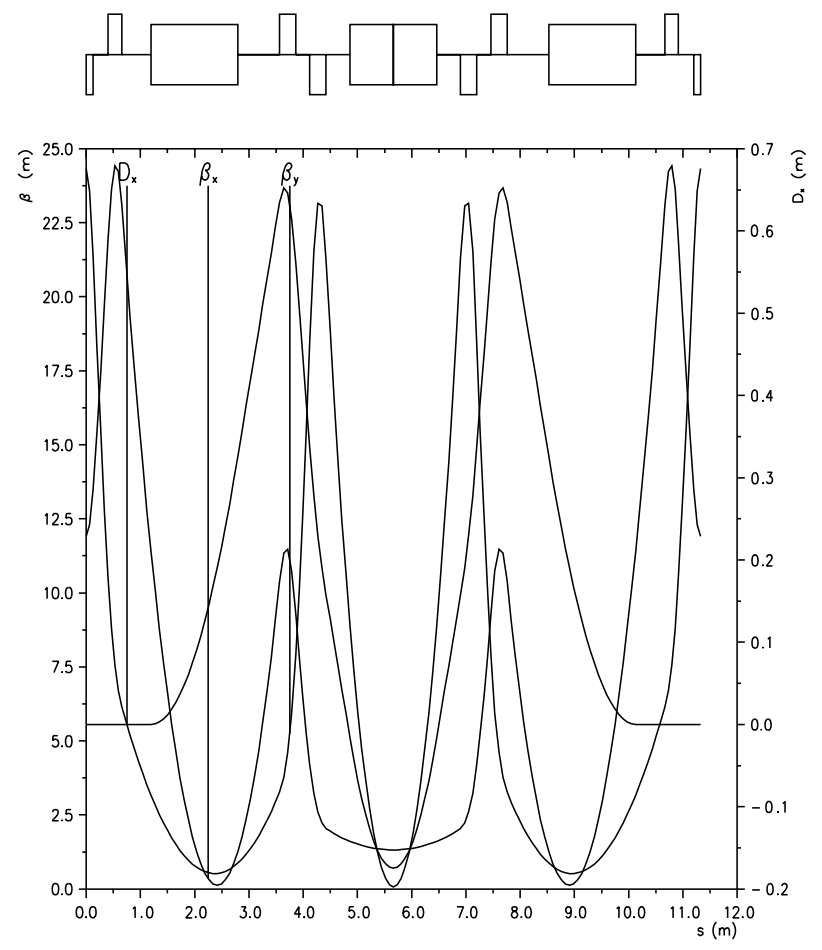

FIG. 2. $90^{\circ}$ isochronous arc for the CLIC $\mathrm{rf}$ power source.

\section{B. Isochronicity-tunable achromat}

It was shown in [5] that it is possible to tune $R_{56}$ between a minimum value (negative) $R_{56, \min }$ and a maximum value (positive) $R_{56, \max }$ without displacing the quadrupoles of this insertion. Thus the lengths of the first two drift spaces $L_{1}$ and $L_{2}$ are fixed and the normalized strengths $k_{1}$ and $k_{2}$ can be expressed as functions of $R_{56}$, which implies inverting the two equations (4). Unfortunately, these are transcendental equations and no close form can be obtained for $k_{1}$ and $k_{2}$. However, it can be shown that it is possible in the thin lens approximation, that is for $l_{q}$ sufficiently small, to fulfill

$$
C_{1}=C_{2}=1, \quad S_{1}=l_{q} \sqrt{k_{1}}, \quad S_{2}=l_{q} \sqrt{k_{2}} .
$$

Then, the absolute values of the focal lengths $f_{1}=l_{q} k_{1}$ and $f_{2}=l_{q} k_{2}$ replace $q_{1}$ and $q_{2}$, respectively. In order to design an $R_{56}$ tunable module it is necessary to find the ranges of $L_{1}, L_{2}, L_{3}$ such that $f_{1}$ and $f_{2}$ remain positive when $R_{56}$ varies from $R_{56, \text { min }}<0$ to $R_{56, \text { max }}>0$.

Let $x_{\min }$ and $x_{\max }$ be defined by

$$
\begin{aligned}
& x_{\min }=\frac{R_{56, \min }}{2}-l_{m}\left(\frac{3}{2}-\frac{\sin \phi_{1}}{\phi_{1}}\right)<0, \\
& x_{\max }=\frac{R_{56, \text { max }}}{2}-l_{m}\left(\frac{3}{2}-\frac{\sin \phi_{1}}{\phi_{1}}\right) .
\end{aligned}
$$

The valid ranges of the lengths $L_{1}, L_{2}$, and $L_{3}$ can be determined by eight sets of conditions. The algebra to obtain them is tedious and can be found in [5]. Hereafter, the results are summarized.

First set:

$$
\begin{gathered}
\frac{\sin \phi_{1}}{\phi_{1}}+\frac{\tan \left(\phi_{2} / 2\right)}{\phi_{2}}<\frac{3}{2}, \quad x_{\max }<-\rho_{2} \tan \left(\phi_{2} / 2\right), \\
L_{3}<\frac{\rho_{2}^{2}}{x_{\max }}+\rho_{2} \cot \left(\phi_{2} / 2\right), \quad-\mathcal{L}_{3}-\frac{d \rho_{2} \sin \phi_{1}}{x_{\min }}<L_{2}<-\mathcal{L}_{3}, \\
L_{1}<-d-\frac{x_{\min }\left(L_{2}+\mathcal{L}_{3}\right)}{\rho_{2} \sin \phi_{1}} .
\end{gathered}
$$


Second set:

$$
\begin{gathered}
\frac{\sin \phi_{1}}{\phi_{1}}+\frac{\tan \left(\phi_{2} / 2\right)}{\phi_{2}}<\frac{3}{2}, \quad x_{\max }<-\rho_{2} \tan \left(\phi_{2} / 2\right) \\
L_{3}<\frac{\rho_{2}^{2}}{x_{\max }}+\rho_{2} \cot \left(\phi_{2} / 2\right), \quad L_{2}>-\mathcal{L}_{3}, \\
L_{1}<-d-\frac{x_{\max }\left(L_{2}+\mathcal{L}_{3}\right)}{\rho_{2} \sin \phi_{1}}
\end{gathered}
$$

Third set:

$$
\begin{gathered}
x_{\max }<\rho_{2} \sin \phi_{1}, \quad \frac{\rho_{2}^{2}}{x_{\min }}+\rho_{2} \cot \left(\phi_{2} / 2\right)<L_{3}<\rho_{2} \cot \left(\phi_{2} / 2\right), \\
L_{2}<-\mathcal{L}_{3}, \quad L_{1}>-d-\frac{x_{\max }\left(L_{2}+\mathcal{L}_{3}\right)}{\rho_{2} \sin \phi_{1}}
\end{gathered}
$$

Fourth set:

$$
\begin{aligned}
& x_{\max }>\rho_{2} \sin \phi_{1}, \quad \frac{\rho_{2}^{2}}{x_{\min }}+\rho_{2} \cot \left(\phi_{2} / 2\right)<L_{3}<\rho_{2} \cot \left(\phi_{2} / 2\right), \\
& L_{2}<-\mathcal{L}_{3} \frac{x_{\max }-x_{\min }}{\rho_{2} \sin \phi_{1}-x_{\min }}, \quad L_{1}>-d-L_{2}-\mathcal{L}_{3} \frac{x_{\max }}{\rho_{2} \sin \phi_{1}} .
\end{aligned}
$$

Fifth set:

Sixth set:

$$
\begin{gathered}
x_{\max }<\rho_{2} \sin \phi_{1}, \quad \frac{\rho_{2}^{2}}{x_{\min }}+\rho_{2} \cot \left(\phi_{2} / 2\right)<L_{3}<\rho_{2} \cot \left(\phi_{2} / 2\right), \\
L_{2}>-\mathcal{L}_{3}, \quad L_{1}>-d-\left(L_{2}+\mathcal{L}_{3}\right) \frac{x_{\min }}{\rho_{2} \sin \phi_{1}} .
\end{gathered}
$$

$$
\begin{gathered}
x_{\max }>\rho_{2} \sin \phi_{1}, \quad \frac{\rho_{2}^{2}}{x_{\min }}+\rho_{2} \cot \left(\phi_{2} / 2\right)<L_{3}<\rho_{2} \cot \left(\phi_{2} / 2\right), \\
L_{2}>-\mathcal{L}_{3} \frac{x_{\max }-x_{\min }}{\rho_{2} \sin \phi_{1}-x_{\min }}, \quad L_{1}>-d-\left(L_{2}+\mathcal{L}_{3}\right) \frac{x_{\min }}{\rho_{2} \sin \phi_{1}} .
\end{gathered}
$$

Seventh set:

$$
x_{\max }<0, \quad L_{3}>\rho_{2} \cot \left(\phi_{2} / 2\right), \quad L_{1}>-d-\frac{\left(L_{2}+\mathcal{L}_{3}\right) x_{\min }}{\rho_{2} \sin \phi_{1}} .
$$

Eighth set:

$$
\begin{gathered}
x_{\max }>0, \quad \rho_{2} \cot \left(\phi_{2} / 2\right)<L_{3}<\frac{\rho_{2}^{2}}{x_{\max }}+\rho_{2} \cot \left(\phi_{2} / 2\right), \\
L_{1}>-d-\frac{\left(L_{2}+\mathcal{L}_{3}\right) x_{\min }}{\rho_{2} \sin \phi_{1}},
\end{gathered}
$$

with the following definitions:

$$
\begin{aligned}
\mathcal{L}_{3} & =L_{3}-\rho_{2} \cot \left(\phi_{2} / 2\right), \\
d & =l-\frac{\rho_{2}}{\sin \phi_{1}} .
\end{aligned}
$$

This approach has been used to obtain a preliminary design of the transfer line between the delay loop and the combiner ring of the test facility to be built at CERN to validate the concept retained for the production of the $30 \mathrm{GHz}$ rf power needed by the CLIC two beam scheme [3]. The transfer line should be able to increase or decrease the bunch length by $1.6 \mathrm{~mm}$. Given the $\Delta p / p$ of the order of $1 \%$, the range of $R_{56}$ is then between -0.16 and $0.16 \mathrm{~m}$. To accommodate this transfer line in an " $\mathrm{S}$ " shape inside the available space, it is made of two insertions, one bending the beam by $75^{\circ}$ and the other bending it 


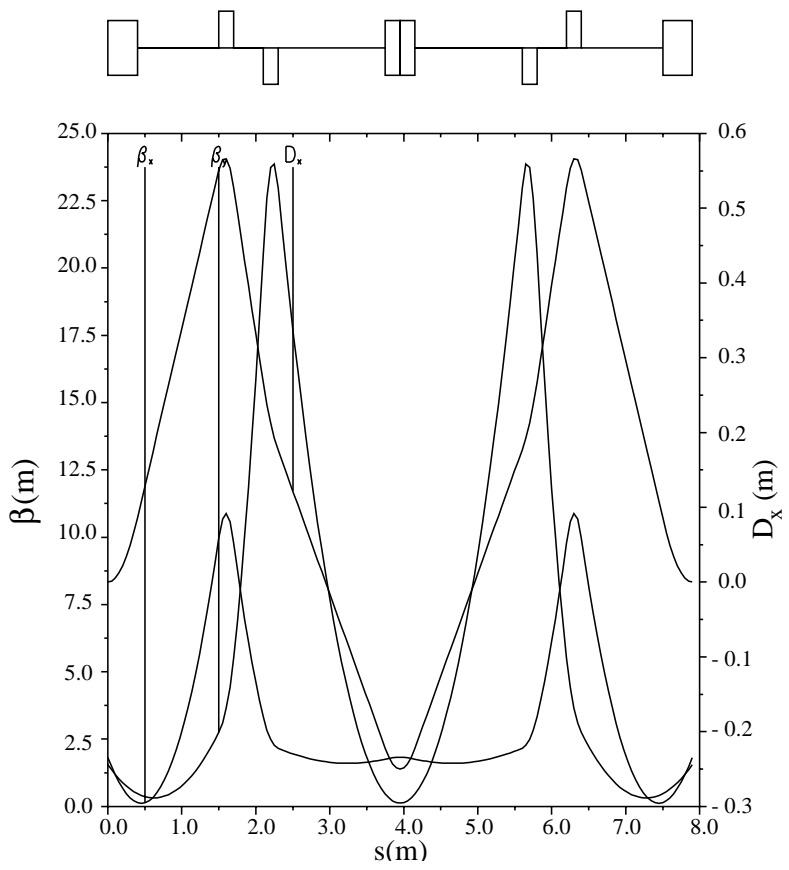

FIG. 3. Optical functions for $R_{56}=-0.04 \mathrm{~m}$.

back by $-75^{\circ}$. A range of possible solutions has been identified without using numerical searches which are very unstable in this specific problem. Thus the insertion could be optimized to find a compromise between the overall length imposed by the building dimensions and the optics (Twiss parameters). The most useful set of condi-
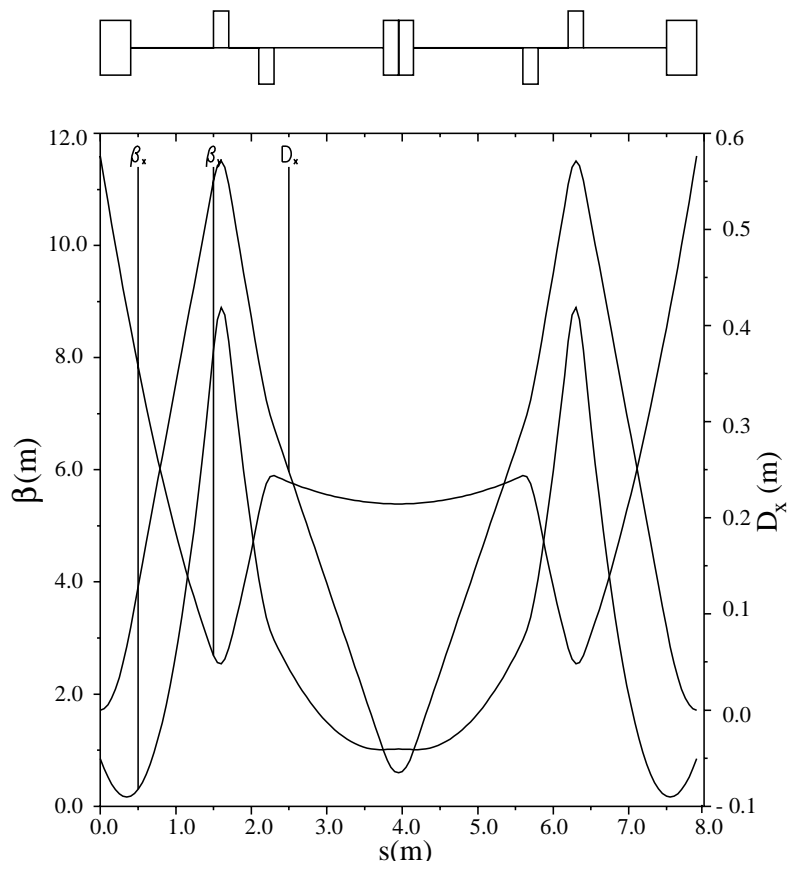

FIG. 4. Optical functions for $R_{56}=0 \mathrm{~m}$.

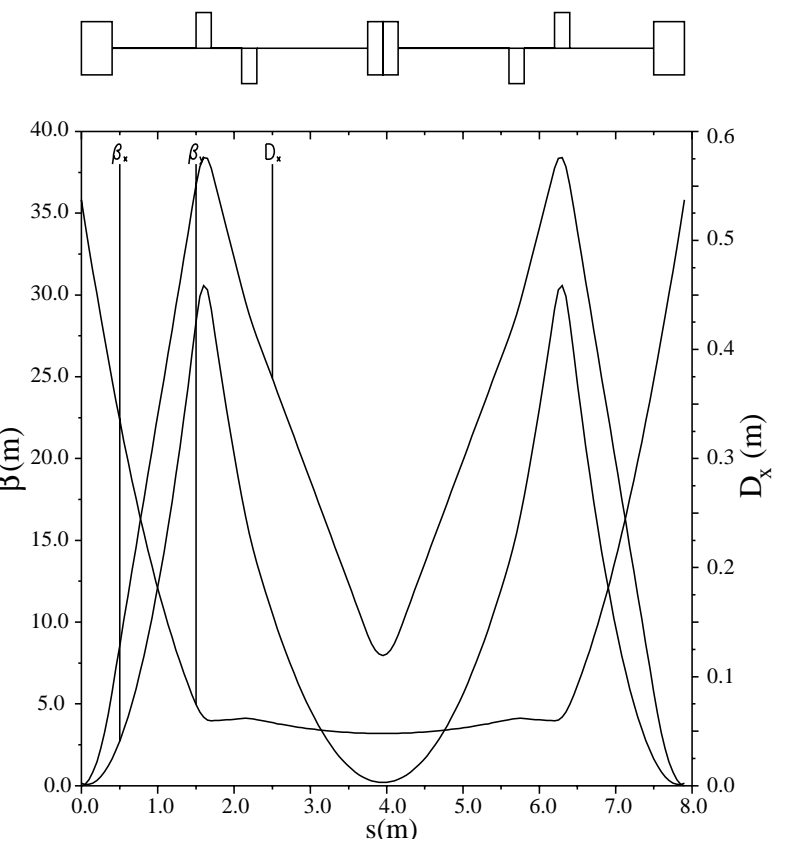

FIG. 5. Optical functions for $R_{56}=0.04 \mathrm{~m}$.

tions in the design of this transfer line has been the third. The thick lens results have been easily obtained through the standard program MAD [1] by setting the quadrupole length at the nominal value of $0.2 \mathrm{~m}$ and using the thin lens data as initial conditions. The three dipoles of the selected insertion have the same length $(0.4 \mathrm{~m})$ and generate the same beam deflection $\left(25^{\circ}\right)$. The drift lengths are $L_{1}=$ $1.2 \mathrm{~m}, L_{2}=0.6 \mathrm{~m}$, and $L_{3}=1.55 \mathrm{~m}$.

Figures 3-5 show the optical functions of the full insertion when the $R_{56}$ parameter of half of one single insertion is $-0.04,0$, and $0.04 \mathrm{~m}$, respectively.

For a beam energy of $400 \mathrm{MeV}$, the gradients of the first and second quadrupoles vary between 12.04 and $7.81 \mathrm{~T} / \mathrm{m}$, and between 12.13 and $1.29 \mathrm{~T} / \mathrm{m}$, respectively. They are shown in Fig. 6.

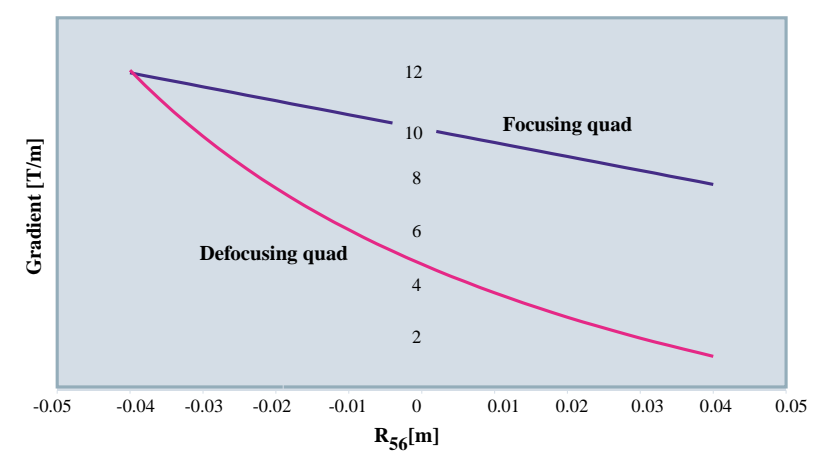

FIG. 6. (Color) Quadrupole gradients at $400 \mathrm{MeV}$. 


\section{Symmetric matching triplet}

The special insertions presented above are usually part of transfer lines or rings. The connection between them is done by matching sections. To avoid large excursions of the betatron functions, the easiest way is to take advantage of the insertion symmetry and to ensure that the values of the Twiss parameters are the same at both ends. In order to reduce the contribution of magnetic errors and sextupole effects to a minimum, the phase advance over a small number of insertions should be as close as possible to an integer multiple of $\pi$ in both planes. The symmetric triplet shown in Fig. 7 is the insertion with the smallest number of components which provides the required degrees of freedom.

It has four free variables (the two drift lengths $l_{1}, l_{3}$ and the magnetic gradients $G_{2}, G_{4}$, respectively, of the first and one-half of the second quadrupole). It can easily be proved [6] that the diagonal elements of the transfer matrix for each plane are all the same. Thus the number of independent values which can be fixed by selecting the Twiss parameters at the entrance and at the exit of the triplet is also 4. The number of solutions is finite and does not depend upon additional assumptions. Moreover, closed form solutions can be obtained in the thin lens approximation. The equality of the diagonal elements of the transfer matrix for each plane forces an important constraint on the betatron function in that plane at the entrance and at the exit of a symmetric triplet. Neglecting the special case where the phase advance is an odd multiple of $\pi$, we may distinguish two situations. The first one, which can be called a mirror-symmetry triplet, occurs when the betatron functions at both ends have equal values and their derivatives have opposite values, the phase advance being a free parameter. This is the case of interest for building up a ring with a chain of isochronous modules separated by such matching insertions. The second configuration occurs when the betatron function has different values at each end of the symmetric triplet with its derivatives being free parameters, but in this case the phase advance can no longer be freely chosen and is given by

$$
\cot \mu=\frac{\beta_{2} \alpha_{1}+\beta_{1} \alpha_{2}}{\beta_{1}-\beta_{2}},
$$

where $\alpha_{1}, \beta_{1}$ and $\alpha_{2}, \beta_{2}$ are the Twiss functions at the entrance and the exit of the triplet (see Fig. 7), respectively. Thus we have the choice between only two phase advances which differ by $\pi$. The more general case, which will

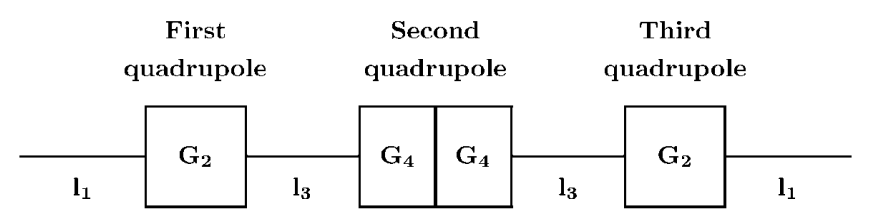

FIG. 7. Schematic of a symmetric triplet. be called a matching triplet, can be used, for example, in matching a FODO line to a special transfer line containing one of the achromats presented above.

In the thin lens approximation, the transfer matrices for the horizontal and vertical planes can be expressed as functions of the two drift lengths $l_{1}, l_{3}$ and the quadrupole strengths $g_{2}=k_{2} l_{q}, g_{4}=k_{4} l_{q}$, where $l_{q}$ is the quadrupole length and $k_{2}, k_{4}$ are the normalized gradients. In this case, the general solution is characterized by [6]

$$
\begin{aligned}
\ell_{1} & =\frac{1}{c^{2}-d^{2}}[(b c-a d) z-(b d-a c)], \\
g_{2} & =\frac{c^{2}-d^{2}}{(b c-a d)\left(1-z^{2}\right)}, \\
\ell_{3} & =\frac{(b c-a d)\left(1-z^{2}\right)}{z\left(c^{2}-d^{2}\right)}, \\
g_{4} & =\frac{z^{2}(d-c z)}{1-z^{2}}
\end{aligned}
$$

where $z$ is a solution of the cubic equation

$$
z^{3}-\frac{2 d}{c} z^{2}+z+\frac{d^{2}-c^{2}}{c(b c-a d)}=0,
$$

and $a, b, c, d$ are defined by

$$
\begin{gathered}
a=\left(t_{h, 11}+t_{v, 11}-2\right) / 4, \quad b=\left(t_{h, 11}-t_{v, 11}\right) / 4, \\
c=\left(t_{h, 21}+t_{v, 21}\right) / 4, \quad d=\left(t_{h, 21}-t_{v, 21}\right) / 4,
\end{gathered}
$$

where $t_{h, n m}$ and $t_{v, n m}$ are the elements of the horizontal and vertical transfer matrices, respectively. It is assumed that $b c-a d \neq 0$, and the trivial cases of $c=0$ or $c= \pm d$ are neglected. Of course, only real values of $z$ leading to positive lengths of drift spaces are retained. The extension to thick lenses is easily obtained by solving for the normalized gradients $k_{2}, k_{4}$ from the two nonlinear equations [6] or by a standard numerical matching program such as MAD.

\section{CONCLUDING REMARKS}

The aim of this paper is to show how important it is to develop analytical treatments and not to rely only on numerically driven programs for insertion designs. Analytical approximations provide sure guidelines and a very useful insight of the main features of the problem. Moreover, they give good starting points which not only speed up the numerical search but are often crucial for convergence, especially when the parameter hyperspace is not smooth in the neighborhood of the required solution. The latter is frequently not unique, and the choice is based on additional constraints such as the maximum excursion of the Twiss parameters or geometrical conditions which are often mutually conflicting. Thus a compromise has to be made which cannot be automated inside a program 
but necessitates the active intervention of the user, which requires interactive capabilities and an extensive array of tools, including graphics, to aid the designer. Until now, the programs available are implemented for historical reasons in FORTRAN 77 running in a UNIX environment. A consolidation is needed to obtain a fully portable product. At the same time, the routines should be streamlined and standardized by moving to an object-oriented language.
[1] H. Grote and F.C. Iselin, CERN Report No. CERN/ SL/90-13(AP) (Rev. 4), 1995; http://www.wolfram.com/ maeder/ProgInMath/.

[2] The CLIC Study Team, CERN Report No. CERN 2000008, 2000, edited by G. Guignard.

[3] The CLIC Study Team, CERN CLIC Note No. 402, 1999.

[4] E. T. d'Amico and G. Guignard, CERN Report No. CERN/ SL/95-120, 1995.

[5] E. T. d'Amico, CERN CLIC Note No. 459, 2000.

[6] E. T. d'Amico, CERN CLIC Note No. 322, 1997. 